\title{
EDITORIAL
}

\section{IN THE JUNE 2006 ISSUE OF CLINICS}

\author{
Mauricio Rocha-e-Silva, Editor
}

In this issue of Clinics, we highlight a paper by Nakano et al, who describe a new stress test using isokinetic dynamometry for the evaluation of the functional limitation caused by occlusive arterial disease in upper limbs. Twenty three patients with subclavian arterial occlusion were compared to 23 patients of similar age exhibiting non-occlusive arterial pathologies. This test allows for a better choice of therapeutic approach for each and report on the objective parameters that allow comparisons of the results of different treatments, including for long-term follow-up. Classification of patients according to their functional impairment was also improved.

Sircili et al retrospectively studied anatomical and functional outcomes of feminizing genitoplasty in 34 patients with virilizing congenital adrenal hyperplasia and found that single-stage feminizing genitoplasty-vulvoplasty, clitoroplasty, and Y-V perineal flap_produces good cosmetic and functional results, and that the surgical approach prevents the need for neovaginoplasty even in patients with high vaginal insertion.

Macchetti et al demonstrate the association of the immunophenotype of tumor-infiltrating lymphocytes in early breast cancer with the spread of tumor cells to axillary lymph nodes, by analyzing tumor samples from 23 patients from the Department of Gynecology and Obstetrics of the Ribeirão Preto Medical School. They conclude that the association of tumor-infiltrating $\mathrm{CD}^{+} \mathrm{T}$ lymphocytes with lymph node metastasis suggests a role for these cells in the spread of neoplasia to lymph nodes in patients with early breast cancer.

Ruano et al review data from 38 cases of prenatally diagnosed congenital diaphragmatic hernia - gestational age at diagnosis, fetal karyotyping, side of diaphragmatic defect,

Hospital das Clínicas, São Paulo University Medical School - São Paulo/ SP, Brazil.

Email: mrsilva36@hcnet.usp.br presence of associated structural malformations, hepatic herniation, and severe mediastinal shift - and find that perinatal mortality was very high in spite of the great advances in prenatal and postnatal diagnosis and management.

Tiseo et al compare the effects of traditional nonsteroidal anti-inflammatory drugs and nonsteroidal anti-inflammatory drugs that are selective cyclooxygenase-2 inhibitors in the process of bone regeneration in 44 Wistar-strain rats. Fracture consolidation occurred within less than 2 weeks and the use of nonsteroidal anti-inflammatory drugs did not significantly influence this process.

Aquino et al assessed the reliability and validity of isokinetic dynamometry in the qualitative determination of the evolution of total knee arthroplasty by analyzing 20 patients undergoing the procedure and 25 controls. This study shows that the detection of deficiencies in the balance of flexor and extensor muscles allows the selection of specific rehabilitation exercises according to the individual needs of each surgical patient.

Haddad et al analyze age, diagnosis, anatomic site of the ocular injury, visual acuity, and prescription of optical aids for 385 children and adolescents attending the Ophthalmologic Clinic's Low Vision Service at the São Paulo University Medical School. They find that there is a need for prevention of primary, secondary, and tertiary visual impairment and that the use of optical aids by school age children will contribute to their social inclusion.

Rocha Filho et al report that hypertonic saline solution can be used as an adjunct in the neuroprotective strategy during orthotopic liver transplantation for fulminant hepatic failure, reducing intracranial pressure while restoring arterial blood pressure and promoting sustained increase in the cerebral perfusion pressure.

Park et al review the use of noninvasive mechanical ventilation in patients with acute cardiogenic pulmonary edema, and Wiernsperger et al review obstructive sleep apnea. We also publish 5 letters to the Editor. 